\title{
Neonatal bilirubin production, reflected by carboxyhaemoglobin concentrations, in Down's syndrome
}

\author{
Michael Kaplan, Hendrik J Vreman, Cathy Hammerman, David K Stevenson
}

\begin{abstract}
Aim-To determine whether increased bilirubin production, reflected by blood carboxyhaemoglobin ( $\mathrm{COHb}$ ) values, is responsible for hyperbilirubinaemia in cases of Down's syndrome with no obvious cause for excessive jaundice.

Methods-Blood was sampled on the third day of life for COHb, total haemoglobin (tHb), and serum total bilirubin, from 19 consecutively born neonates with Down's syndrome (a subset of 34 term babies), who had developed hyperbilirubinaemia (serum bilirubin $\geqslant 256 \mu \mathrm{mol}$ ), and from 32 term controls. $\mathrm{COHb}$, measured by gas chromatography, was corrected for inspired CO (COHbc) and expressed as a percentage of $\mathrm{tHb}$.
\end{abstract}

Results-Significantly more of the Down's syndrome subset developed hyperbilirubinaemia than the controls $(10 / 19(52 \%)$ vs $7 / 32(22 \%)$, relative risk $2.4,95 \%$ confidence intervals (CI) 1.10 to 5.26). Third day serum bilirubin values (mean (SD)) were higher in the Down's syndrome neonates than in controls $(214 \pm 63 \mu \mathrm{mol} / 1$ vs $172 \pm 54 \mu \mathrm{mol} / 1$, respectively, $\mathrm{p}=0.015)$. Mean (SD) COHbc values were significantly higher in the Down's syndrome neonates than in controls $(0.92 \pm 0.24 \%$ vs $0.63 \pm 0.17 \%$; $<<0.0001)$. However, Down's syndrome neonates who became hyperbilirubinaemic had similar COHbc values to those who did not $(0.87 \pm 0.26 \%$ and $0.95 \pm$ $0.23 \%$, respectively). These values contrast with those of the controls, in whom a significant increase in COHbc was associated with hyperbilirubinaemia $(0.74 \pm$ $0.15 \%$ vs $0.60 \pm 0.16 \%$, respectively; $\mathbf{p}<0.05)$. tHb values were similar in both groups.

Conclusions-Down's syndrome neonates had a greater risk of hyperbilirubinaemia, and higher COHbc values, than controls. However, excessive bilirubin production could not be exclusively responsible for the hyperbilirubinaemia. By inference, decreased bilirubin elimination probably plays a greater part in its pathogenesis than in controls. Down's syndrome neonates may have abnormal erythropoiesis, leading to increased haem turnover. (Arch Dis Child Fetal Neonatal Ed 1999;81:F56-F60)

Keywords: Down's syndrome; carboxyhaemoglobin; carbon monoxide; jaundice; bilirubin production; haemolysis
Down's syndrome is frequently complicated by neonatal hyperbilirubinaemia. However, the aetiology of this jaundice has not been evaluated. This study aimed to determine the incidence of hyperbilirubinaemia in a cohort of Down's syndrome neonates, and to study the role of bilirubin production in the pathogenesis of this jaundice. As most carbon monoxide (CO) produced in the body derives from the catabolism of haem in equimolar quantities with bilirubin, accurate determination of blood carboxyhaemoglobin $(\mathrm{COHb})$ and correction for inspired $\mathrm{CO}(\mathrm{COHbc})$ can be used as an index of bilirubin production.

\section{Methods}

To determine the incidence of hyperbilirubinaemia in a cohort of Down's syndrome neonates, the clinical courses of term, otherwise healthy, Down's syndrome neonates, born at the Shaare Zedek Medical Center between 1989 and mid-1998, were reviewed. Cases before 1994 were evaluated by retrospective chart review, while cases born after this date were studied as they occurred. All were trisomy 21 by karyotype determination. Neonates who had any obvious cause for jaundice, such as Coombs' positive haemolytic anaemia, cephalohaematoma, maternal diabetes, sepsis, or glucose-6-phosphate dehydrogenase (G-6-PD) deficiency, were excluded from this survey. Routine clinical management included encouragement of breastfeeding, daily observation for the development of jaundice, and serum bilirubin determinations in the babies who become clinically jaundiced. As we could not justify performing daily serum bilirubin tests on babies who were only minimally jaundiced, we combined clinical judgment with laboratory evaluation. However, our medical/nursing staff are especially aware of the high risk of these neonates developing hyperbilirubinaemia, and serum total bilirubin determinations were ordered liberally whenever a Down's syndrome neonate became even moderately jaundiced. Neonates were admitted for a minimum of 72 hours. Discharge was delayed if the serum total bilirubin values were approaching phototherapy criteria at 72 hours, or if phototherapy was required.

Follow up after discharge and subsequent serum total bilirubin determinations were performed, as necessary, on an outpatient basis until it was clear that serum total bilirubin values had stabilised or were decreasing. The compliance rate in our population was excellent, and it is extremely unlikely that the serum 
total bilirubin values could have exceeded 256 $\mu \mathrm{mol} / 1$ without our being aware of it. Phototherapy was started in hospital in those neonates whose serum total bilirubin values exceeded $256 \mu \mathrm{mol} / 1$. Clinical details such as birthweight, gestational age, delivery route, and method of feeding were recorded, as well as the highest serum total bilirubin noted during the first week of life. The maximal serum total bilirubin values were probably attenuated by the institution of phototherapy in the hyperbilirubinaemic babies, and thus did not allow these values to reach their natural peak.

The study protocol was approved by the Institutional Review Board of the Shaare Zedek Medical Center. Blood $\mathrm{COHb}$ and total haemoglobin $(\mathrm{tHb})$ determinations were performed on consecutively born, term, otherwise healthy, Down's syndrome neonates born at the same institution between July 1994 and April 1998. These neonates were a subset of the larger Down's syndrome cohort above and were managed in an identical way. The control group consisted of consecutive, healthy, term neonates born at the same institution at the approximate projected halfway point of the study (January 1996). Between 48 and 72 hours of life, before the institution of phototherapy in all cases, blood for $\mathrm{COHb}$ and $\mathrm{tHb}$ determinations was collected into custom prepared capillary tubes containing heparin, as described before. ${ }^{2}$ The blood samples were stored at $4^{\circ} \mathrm{C}$ and shipped on ice to Stanford University. At the same time as $\mathrm{COHb}$ and $\mathrm{tHb}$ sampling, blood was drawn for serum total bilirubin determination, and a sample of room air was collected.

$\mathrm{COHb}$ determinations were performed by a previously described ${ }^{3}$ sensitive and accurate gas chromatographic method, the concentrations expressed as a percentage of $\mathrm{tHb}$. The latter was determined using a manual cyanmethaemoglobin method, also described before. ${ }^{3}$ Within day variation for the gas chromatographic method was $1.0(0.03) \%$ (mean (SD)), with a $3 \%$ coefficient of variation $(n=10)$, and that for between day analysis $1(0.10) \%$, with a $10 \%$ coefficient of variation $(n=9)$. Respective values for $\mathrm{tHb}$ testing by this method were 135 (3.0) $\mathrm{g} / 1(2.2 \%)(\mathrm{n}=10)$ and $132(3.0) \mathrm{g} / 1$ $(2.3 \%)$ for within day and between day analyses, respectively. ${ }^{3}$

All other laboratory testing was performed at the Shaare Zedek Medical Center. The CO content of the room air specimens was determined using a sensitive electrochemical $\mathrm{CO}$ analyser supplied by Stanford University. ${ }^{4}$ Serum total bilirubin was performed using an automated analyser (Astra 8, Beckman Instruments, Brea, CA, USA) with a modified diazo method. The system was calibrated daily using a commercial standard (Liquichek Pediatric Control, Levels 1 and 2, Bio-Rad Laboratories, Anaheim, CA, USA). The same laboratory facilities were used for inpatient and outpatient serum bilirubin determinations. Blood typing, direct Coombs' testing, and G-6-PD screening were performed using routine, standard laboratory techniques.
For the purpose of the study, hyperbilirubinaemia was defined as a serum total bilirubin $>256 \mu \mathrm{mol} / \mathrm{l}$. Measured $\mathrm{COHb}$ values and $\mathrm{CO}$ concentrations in room air were used to calculate $\mathrm{COHbc}$ by a previously derived formula $(\mathrm{COHbc}=$ measured $\mathrm{COHb}-0.17[\mu \mathrm{l} \mathrm{CO} / 1$ room air $]) .{ }^{5}$ By correcting for inspired $\mathrm{CO}$, any daily or seasonal variation in $\mathrm{CO}$ exposure should have been offset. Continuous variables were compared using Student's $t$ test. Categorical variables were compared using $\chi^{2}$ analysis or Fisher's exact test. The risk of the Down's syndrome subset neonates developing hyperbilirubinaemia, compared with controls, was determined by calculating the relative risk and $95 \%$ confidence intervals (CI), the relative risk of the control group defined as one. Significance was defined either as $\mathrm{p}<0.05$, or a $95 \%$ CI range either wholly above or wholly below 1 .

\section{Results}

Of 36 term neonates with Down's syndrome consecutively born between 1989 and 1998, one with direct Coombs' positive ABO isoimmunisation, and one with both G-6-PD deficiency and maternal diabetes, were excluded from the survey. Therefore, a total of 34 Down's syndrome neonates were reviewed. The mean (SD) gestational age of these neonates was 39 (1) weeks, and birthweight 2956 (367) g. The male:female ratio was 12:22. Seventeen were being breastfed. Nineteen of the $34(55.9 \%)$ Down's syndrome neonates developed a peak serum total bilirubin $>256 \mu \mathrm{mol} / 1$ during the first week of life. The mean maximal serum total bilirubin of the hyperbilirubinaemic neonates was 284 (20) $\mu \mathrm{mol} / 1$, although institution of phototherapy in 18 of these probably modified the peak serum bilirubin values. Serum total bilirubin values in the one infant who did not receive phototherapy peaked at $256 \mu \mathrm{mol} / \mathrm{l}$. Age at the maximal noted serum total bilirubin in the hyperbilirubinaemic Down's syndrome neonates was 77 (21) hours. Gestational age and birthweight of the subgroup of Down's syndrome neonates who developed hyperbilirubinaemia were not significantly different from the entire cohort (38.9 (1.0) weeks and 2900 (374 g), respectively). Only one neonate in this subgroup had a gestational age $<38$ weeks. Fifty three per cent of the hyperbilirubinaemic babies were breastfeeding.

Clinical details for the subset of consecutively born Down's syndrome neonates in whom $\mathrm{COHb}$ determinations were performed, and 32 controls, are summarised in table 1 . Twenty Down's syndrome neonates were studied, but as the COHbc value of one of these neonates was exceptionally high $(3.75 \%$, equivalent to mean $\pm 10 \mathrm{SD}$ ), with a simultaneously drawn serum total bilirubin value of 171 $\mu \mathrm{mol} / \mathrm{l})$, this baby was regarded as an outlier and excluded from the analysis. The Down's syndrome subset therefore includes 19 neonates. Table 1 shows that the risk of neonates of this Down's syndrome subset developing hyperbilirubinaemia was significantly increased compared with controls. All 10 Down's 
Table 1 Clinical details pertaining to subset of Down's syndrome and control neonates for whom $\mathrm{COHbc}$ and $\mathrm{tHb}$ determinations were obtained

\begin{tabular}{llll}
\hline & $\begin{array}{l}\text { Down's } \\
\text { syndrome } \\
n=19\end{array}$ & Controls $n=32$ & Significance \\
\hline Parameter & $2904(312)$ & $3172(436)$ & $\mathrm{p}=0.02$ \\
\hline Birth weight $(\mathrm{g})$ & $38.6(1.1)$ & $39.3(2.1)$ & $\mathrm{NS}$ \\
Gestational age (weeks) & $19(100) \%$ & $28(88) \%$ & $\mathrm{NS}$ \\
Vaginal delivery (n) & $8(42) \%$ & $23(72) \%$ & $\mathrm{NS}$ \\
Breastfed (n) & & $7(22) \%$ & Relative risk $2.4^{\star}$ \\
Serum total bilirubin $\geqslant 256 \mu \mathrm{mol} / \mathrm{l}(\mathrm{n})$ & $10(52.6) \%$ & & $95 \%$ CI: 1.10 to 5.26 \\
& & &
\end{tabular}

Data supplied as mean (SD), or as percentage, as appropriate.

NS: no significant difference.

${ }^{\star}$ Relative risk of Down's syndrome subset compared with control population, the latter defined as one.

Table 2 Day 3 studies (serum total bilirubin, $t \mathrm{Hb}$, and $\mathrm{COHbc}$ ) performed on subset of Down's syndrome and control neonates

\begin{tabular}{llll}
\hline Parmeter & $\begin{array}{l}\text { Down's } \\
\text { syndrome } \\
n=19\end{array}$ & Controls $n=32$ & Significance \\
\hline Serum total bilirubin $(\mu \mathrm{mol} / \mathrm{l})$ & $214(63)$ & $172(54)$ & $\mathrm{p}=0.015$ \\
tHb $(\mathrm{g} / \mathrm{l})$ & $211(25)$ & $202(20)$ & $\mathrm{NS}$ \\
$\mathrm{COHbc}(\%$ of $\mathrm{tHb})$ & $0.92(0.24)$ & $0.63(0.17)$ & $\mathrm{p}<0.0001$ \\
\hline
\end{tabular}

Data supplied as mean (SD).

NS: No significant difference.

syndrome neonates and six of the seven controls who became hyperbilirubinaemic received phototherapy.

Results of the third day studies (serum total bilirubin, $\mathrm{tHb}$, and $\mathrm{COHbc}$ ) are detailed in table 2. Both $\mathrm{COHbc}$ (fig 1) values and serum total bilirubin values were significantly higher in the Down's syndrome neonates than in the controls. However, despite higher $\mathrm{COHbc}$ values, not all of the Down's syndrome neonates developed hyperbilirubinaemia. Furthermore, Down's syndrome neonates with maximal serum total bilirubin values $>256$ $\mu \mathrm{mol} / 1$ did not necessarily have higher $\mathrm{COHbc}$ values than those who did not become hyperbilirubinaemic $(0.87 \pm 0.26 \%$ and $0.95 \pm$ $0.23 \%$, respectively, NS). In contrast, higher $\mathrm{COHbc}$ values were found in those control neonates who developed hyperbilirubinaemia than those who did not $(0.74 \pm 0.15 \%$ vs 0.60 $\pm 0.16 \%$, respectively, $\mathrm{p}<0.05)$.

$\mathrm{COHb}$ values above the median were not a prerequisite for the development of hyperbilirubinaemia in either group: of the Down's syndrome neonates, four of the nine who developed hyperbilirubinaemia had $\mathrm{COHbc}$

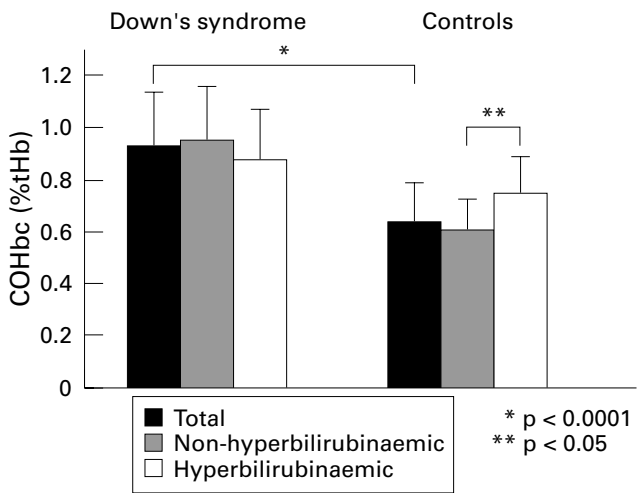

Figure 1 Day 3 mean (SD) COHBc values in Down's syndrome $(n=19)$ and control neonates $(n=32)$ showing values for total population studied, values for those who became hyperbilirubinaemic, and values for those who did not develop hyperbilirubinaemia. values below the median value of $0.89 \%$, while two of the seven hyperbilirubinaemic control neonates had $\mathrm{COHbc}$ values below the median value of $0.60 \%$ for that group. The increased rate of haemolysis had no effect on the $\mathrm{tHb}$ values.

Clinical confounding variables known to delay bilirubin conjugation and excretion were analysed. Birthweight and gestational age were not significantly different when Down's syndrome neonates who developed serum bilirubin values $\geqslant 256 \mu \mathrm{mol} / 1$ were compared with those whose values did not exceed this value $(2877 \pm 384 \mathrm{~g}$ vs $2942 \pm 197 \mathrm{~g}$, and $38.8 \pm$ 1.6 weeks vs $38.6 \pm 0.9$ weeks, respectively). Only one neonate (in the hyperbilirubinaemic group) had a gestational age below 38 weeks. Six of $10(60 \%)$ of the hyperbilirubinaemic group $v s$ three of nine $(33 \%)$ of the nonhyperbilirubinaemic babies were being breastfed (NS). No infant had a weight loss greater than $10 \%$ of birthweight documented in the first three days of life.

\section{Discussion}

An association between Down's syndrome and prolonged and sometimes severe neonatal jaundice was noted by Zuelzer and Brown ${ }^{6}$ and Panizon. ${ }^{7}$ However, subsequent clinical studies and reviews have not related in any detail to the phenomenon of Down's syndrome associated neonatal jaundice. ${ }^{8}$ The more than 55\% incidence of neonatal jaundice in our Down's syndrome cohort reaffirms that neonatal jaundice is common in that condition, and occurs with a frequency not encountered in normal neonatal population groups. ${ }^{9}$ For comparison, the incidence of hyperbilirubinaemia, using the same definition as was used in this study, in a cohort of G-6-PD normal neonates recently reported from the same nursery as these Down's syndrome neonates, was $9.2 \% .^{10}$ The higher incidence in the small group used as controls for $\mathrm{COHbc}$ determinations may have been an artefact due to the low number of babies included in this subset.

The methods used for determination of $\mathrm{COHb}$ have been perfected at Stanford University and assays of both $\mathrm{CO}$ and $\mathrm{tHb}$ have been shown to be extremely sensitive and accurate. $^{211}{ }^{12}$ Blood samples remain stable for up to several months when stored or transported at $4^{\circ} \mathrm{C} .{ }^{2}$ Due to its low detection limit $(0.005 \% \mathrm{COHb})$ and its insensitivity to substances known to cause interference in spectrophotometric methods, the gas chromatography system is regarded as the reference method for accurate $\mathrm{COHb}$ determination. ${ }^{13}$ Although other non-haem sources of CO contribute to the endogenous $\mathrm{CO}$ pool, the predominant source of $\mathrm{CO}$ derives from haem catabolism, $80-90 \%$ of which is derived from haemoglobin. ${ }^{14}$ Berk et $a l^{15}$ have shown that the quantitative contribution to total endogenous $\mathrm{CO}$ production from sources other than haem catabolism is small. Therefore, accurately determined $\mathrm{COHb}$ values, corrected for inspired $\mathrm{CO}$, offer a reliable index of the rate of haem catabolism, and by inference, bilirubin production. For comparison, we have provided 
COHbc values for other haemolytic conditions, taken from neonates studied in the same nursery as the current study: G-6-PD deficient neonates who developed hyperbilirubinaemia had a mean third day $\mathrm{COHbc}$ value of $0.80 \pm$ $0.19 \%,{ }^{16}$ while direct Coombs' test positive $\mathrm{ABO}$ isoimmunised neonates ranged from 0.9 $+0.3 \%$ in the milder cases, to $1.8 \pm 0.7 \%$ in the more severe cases. ${ }^{17}$

Serum total bilirubin values at any one time depend on a balance between the rates of bilirubin production and bilirubin elimination. The pathogenesis of neonatal hyperbilirubinaemia is clearly multifactorial, influencing both bilirubin production and elimination. The high $\mathrm{COHbc}$ values documented in the current Down's syndrome cohort reflected an overall increased rate of haem catabolism and therefore bilirubin production. However, despite this increase in haem breakdown, not all Down's syndrome neonates developed hyperbilirubinaemia. Furthermore, in neither the study nor control groups were increased $\mathrm{COHbc}$ values a prerequisite for the development of hyperbilirubinaemia. While there is a tendency to attribute neonatal hyperbilirubinaemia to increased bilirubin production, bilirubin elimination, which was not accounted for in the current study, can vary enormously among patients. This process involves uptake of bilirubin from the serum into hepatocytes, conjugation with glucuronic acid to form direct bilirubin, and excretion via the bile into the intestine. None of these processes is easily evaluated by non-invasive clinical means. Clinical variables known to affect bilirubin metabolism, such as gestational age, breastfeeding, and excessive weight loss, were examined, but were not significantly different when hyperbilirubinaemic and non-hyperbilirubinaemic Down's syndrome babies were compared. Decreased bilirubin conjugation may have played a greater part in the production of the hyperbilirubinaemia in Down's syndrome neonates compared with controls, similar to that recently demonstrated in G-6-PD deficiency. ${ }^{10}$

The reason for the increased bilirubin production is unknown. The defect in bone marrow regulation in Down's syndrome extends to many cellular elements, and varied haematological abnormalities, affecting erythrocyte, granulocyte, and platelet cell lines, have been documented and comprehensively summarised. ${ }^{18}{ }^{19}$ Polycythaemia, very common in Down's syndrome neonates, ${ }^{720}$ and a likely cause of increased haem breakdown, was surprisingly not demonstrated, and cannot be implied, in the present series. Wilson et $a l^{21}$ showed that neonates with Down's syndrome had lower haemoglobin $\mathrm{F}$ concentrations at birth, which decreased more rapidly with age, and higher haemoglobin A2 concentrations, tending to increase more rapidly after birth, than normal controls. There is some evidence of decreased erythrocyte life span and premature ageing of erythrocytes, with increased removal from the circulation, in adults with Down's syndrome. ${ }^{22}{ }^{23}$ As the gene for superoxide dismutase has been located to chromosome 21 , the additional genetic material for this enzyme results in an excess of $\mathrm{Cu} / \mathrm{Zn}$ superoxide dismutase in Down's syndrome ${ }^{24}$ which may also be responsible for premature tissue aging. De Haan et $a l^{25}$ reported an altered superoxide dismutase 1 to glutathione peroxidase ratio in Down's syndrome tissue, and suggested that this imbalance may contribute to the accumulation of $\mathrm{H}_{2} \mathrm{O}_{2}$, with the resultant formation of tissue damaging hydroxyl radicals. Any of the above mentioned factors may contribute to the exaggerated haemolysis and increased bilirubin production evident in our $\mathrm{COHbc}$ studies. Taking into account the many haematological and biochemical abnormalities already associated with Down's syndrome, we speculate that these neonates may also have ineffective erythropoiesis, resulting in increased haem turnover.

This study was supported in part at Stanford University by grant Nos. 14426 and RR00070 from the National Institutes of Health, the Mary L Johnson Research Fund, the Hess Research Fund, and the Providence Foundation.

1 Vreman HJ, Mahoney JJ, Stevenson DK. Carbon monoxide and carboxyhemoglobin. Adv Pediatr 1995;42:303-34.

2 Vreman HJ, Stevenson DK, Zwart A. Analysis for carboxyhemoglobin by gas chromatography and multicomponent spectrophotemetry compared. Clin Chem 1987:33:694-7

3 Vreman HJ, Kwong LK, Stevenson DK. Carbon monoxide in blood: an improved microliter blood-sample collection system, with rapid analysis by gas chromatography. Clin
Chem 1984;30:1382-5.

4 Vreman HJ, Stevenson DK, Oh W, et al. Semiportable electrochemical instrument for determining carbon monoxide in breath. Clin Chem 1994;40:1927-33

5 Ostrander CR, Cohen RS, Hopper AO, Cowan BE, Stevens GB, Stevenson DK. Paired determinations of blood carboxyhemoglobin concentrations and carbon monoxide excretion rate in term and preterm infants. 7 Lab Clin Med 1982;100:745-55.

6 Zuelzer WW, Brown AK. Neonatal jaundice: a review. Am $\mathcal{f}$ Dis Child 1961;101:113-53.

7 Panizon F. Neonatal jaundice in Down's syndrome. Lancet 1965;ii:495.

8 Hayes A, Batshaw ML. Down Syndrome. Pediatr Clin North Am 1993;40:523-35.

9 Maisels MJ. Jaundice. In: Avery GB, Fletcher MA, MacDonald MJ, eds. Neonatology: Pathophysiology and management of the newborn. Philadelphia: JB Lippincott Co., 1994:630-725.

10 Kaplan M, Renbaum P, Levy-Lahad E, Hammerman C, Lahad A, Beutler E. Gilbert syndrome and glucose-6phosphate dehydrogenase deficiency: A dose-dependent genetic interaction crucial to neonatal hyperbilirubinemia. Proc Natl Acad Sci USA 1997;94:12128-32.

11 Mahoney JJ, Vreman HJ, Stevenson DK, Van Kessel AL. Measurement of carboxyhemoglobin and total hemoglobin by five specialized spectrophotometers (COoximeters) in comparison with reference methods. Clin Chem 1993;39:1693-700.

12 Vreman HJ, Stevenson DK. Carboxyhemoglobin determined in neonatal blood with a CO-oximeter unaffected by fetal oxyhemoglobin. Clin Chem 1994;40:1522-7.

13 US Environmental Protection Agency. EPA 600/8-90/045F. Air quality for carbon monoxide. Research Triangle Park, NC: Environmental Criteria and Assessment Office, Office of Health and Environmental Assessment, Office of Research and Development. US Environmental Protection Agency 1991;1-1 to12-23.

14 Rodgers PA, Vreman HJ, Dennery PA, Stevenson DK. Sources of carbon monoxide (CO) in biological systems and applications of CO detection technologies. Semin Perinatol 1994;18:2-10.

15 Berk PD, Rodkey FL, Blaschke TF, Collison HA, Waggoner JG. Comparison of plasma bilirubin turnover and carbon monoxide production in man. F Lab Clin Med 1974;83:2937 .

16 Kaplan M, Vreman HJ, Hammerman C, Leiter C, Abramov A, Stevenson DK. Contribution of haemolysis to jaundice in Sephardic Jewish glucose-6-phosphate dehydrogenase deficient neonates. Br F Haematol 1996;93:822-7.

7 Hammerman C, Kaplan M, Vreman HJ, Stevenson DK. Intravenous immune globulin in neonatal $\mathrm{ABO}$ isoimmunization: factors associated with clinical efficacy. Biol Neonate 1996;70:69-74.

18 Smith GF, Berg JM. Haematology. In: Down's Anomaly. 2nd edn. Edinburgh:Churchill Livingstone, 1976: 100-18.

19 Miller $M$, Cosgriff JM. Hematological abnormalities in newborn infants with Down syndrome. Am $\mathcal{f}$ Med Genet 1983;16:173-7.

20 Weinberger MM, Oleinik A. Congenital marrow dysfunction in Down's syndrome. F Pediatr 1970;77:273-9. 
21 Wilson MG, Schroeder WA, Graves DA, Kach VD. Postnatal change of hemoglobins $\mathrm{F}$ and A2 in infants with Down's trisomy. Pediatrics 1968;42:349-53.

22 Bartosz G, Kedziora J. Aging of the erythrocyte. XX Decreased red cell life span in Down's Syndrome. Cell Biol Int Rep 1982;6:1119-22.

23 Bartosz G, Soszynski M, Kedziora J. Aging of the erythrocyte. VI. Accelerated red cell membrane aging in Down's Syndrome. Cell Biol Int Rep 1982;6: $73-7$.

24 Crosti N, Serra A, Rigo A, Viglino P. Dosage effect of SOD-A gene in 21-trisomic cells. Hum Genet 1976;31:197-202.

25 deHaan JB, Cristiano F, Ianello RC, Kola I. Cu/Zn superoxide dismutase and glutathione peroxidase during aging. Biochem Mol Biol Int 1995;35:1281-97. 